\title{
Avaliação morfométrica da profundidade do complexo forame/canal/sulco infra- orbitário (FIO/CIO/SIO)
}

\author{
Morphometric evaluation of the depth of the \\ forame/canal/groove infra orbitary (FIO/CIO/SIO)
}

\author{
Evaluación morfométrica de la profundidad del \\ forame/canal/groove infra orbitary (FIO/CIO/SIO)
}

\author{
Catarina Alipio de Freitas ${ }^{1}$, Isabella dos Sanches Santos Borges ${ }^{1}$, \\ Gabriel Fernandes de Oliveira ${ }^{1}$, Thomas dos Reis Rodrigues ${ }^{1}$, Fábio \\ César Prosdócimi ${ }^{2}$
}

1. Acadêmico(a) no curso de Medicina da Universidade Meteropolitana de Santos, Santos-SP, Brasil. 2.Professor Doutor docente na Universidade Metropolitana de Santos e orientador, Santos-SP, Brasil.

\begin{abstract}
Resumo
Introdução. O complexo forame infra-orbitário (FIO)/canal infra-orbitário (CIO)/ sulco infraorbitário (SIO) é uma formação anatômica por onde percorre o nervo infra-orbitário (NIO), estrutura essa considerada de importância para abordagem cirúrgica do assoalho orbital em fraturas orbitais do tipo "blow out". Objetivo. Investigar as diferenças morfométricas e a prevalência da profundidade do complexo CIO/SIO em crânios secos, e apontar a importância dessas variações na prática cirúrgica de lesões de nervos e face. Método. Utilizou-se 15 crânios adultos (30 lados), a partir dos quais foram tiradas medidas de profundidade do complexo FIO/CIO/SIO. O estudo foi realizado na Universidade Metropolitana de Santos com o auxílio de um paquímetro digital. Resultados. Observou-se pequenas variações entre as medidas de profundidade, apresentando um valor mínimo de $17,13 \mathrm{~mm}$ e um valor máximo de $37,08 \mathrm{~mm}$. Sendo a média de $27,243 \pm 4,729723 \mathrm{~mm}$. Conclusão. O conhecimento preciso da anatomia da região infra-orbitária é essencial para uma cirurgia mais segura e eficaz de correção de fratura do assoalho de órbita, visando a preservação da visão e da estética. Dessa forma, as complicações pós-operatórias podem ser diminuídas e o melhor resultado pode ser fornecido.
\end{abstract}

Unitermos. Profundidade; complexo; canal infra-orbitário

\begin{abstract}
Introduction. The infra-orbital foramen (FIO)/infra-orbital canal (CIO)/infra-orbital groove (SIO) complex is an anatomical formation through which the infra-orbital nerve (NIO) travels, a structure that is considered important to approach orbital floor surgery in orbital "blow out" fractures. Objective. To investigate the morphometric differences and the prevalence of the depth of the CIO/SIO complex in dry skulls, and to point out the importance of these variations in the surgical practice of nerve and face injuries. Method. 15 adult skulls (30 sides) were used, from which depth measurements of the FIO/CIO/SIO complex were taken. The study was carried out at the Metropolitan University of Santos with the aid of a digital caliper. Results. Small variations were observed among depth measurements, with a minimum value of $17.13 \mathrm{~mm}$ and a maximum value of $37.08 \mathrm{~mm}$. With the average of $27.243 \pm 4.729723 \mathrm{~mm}$. Conclusion. Accurate knowledge of the anatomy of the infraorbital region is essential for a safer and more effective surgery to correct fractures of the orbital floor, with a view to preserving vision and aesthetics. In this way, postoperative complications can be reduced, and the best result can be provided.
\end{abstract}

Keywords. Depth; complex; infra-orbital canal 


\section{Resumen}

Introducción. El complejo foramen infraorbitario (FIO)/canal infraorbitario (CIO)/surco infraorbitario (SIO) es una formación anatómica a través de la cual viaja el nervio infraorbitario (NIO), una estructura que se considera importante para el abordaje quirúrgico del suelo orbitario en fracturas por estallido orbitario. Objetivo. Investigar las diferencias morfométricas y la prevalencia de la profundidad del complejo CIO/SIO en cráneos secos, y señalar la importancia de estas variaciones en la práctica quirúrgica de las lesiones nerviosas y faciales. Método. Se utilizaron 15 cráneos adultos (30 lados), de los cuales se tomaron medidas de profundidad del complejo FIO/CIO/SIO. El estudio se llevó a cabo en la Universidad Metropolitana de Santos con la ayuda de un calibre digital. Resultados. Se observaron pequeñas variaciones entre las medidas de profundidad, con un valor mínimo de $17,13 \mathrm{~mm}$ y

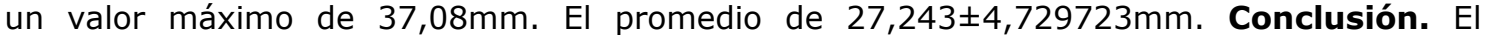
conocimiento preciso de la anatomía de la región infraorbitaria es fundamental para una cirugía más segura y eficaz para corregir las fracturas del suelo orbitario, con el objetivo de preservar la visión y la estética. De esta forma, se pueden reducir las complicaciones postoperatorias y se puede obtener el mejor resultado.

Palabras clave. Profundidad; complejo; canal infraorbital

Trabalho realizado na Universidade Meteropolitana de Santos, Santos-SP, Brasil.

\section{INTRODUÇÃO}

O sulco infra-orbitário estende-se em direção anterior a partir da fissura orbital inferior. Anteriormente, o sulco transforma-se em um canal dentro do seio maxilar, que passa paralelamente ao assoalho da órbita formando por fim o forame infra-orbitário da face superficial do osso maxilar ${ }^{1,2}$. A esse conjunto de estrutura, damos o nome de complexo forame infra-orbitário (FIO)/canal infra-orbitário (CIO)/sulco infra-orbitário (SIO), que são considerados parâmetros cirúrgicos importantes na reparação de fraturas do tipo "blow-out".

O nervo infra-orbitário, que é uma continuação direta do nervo maxilar na órbita, pela fissura orbital inferio, segue um trajeto pelo sulco e no interior do canal infra-orbitário, por fim emerge do crânio pelo forame infra-orbitário para a 
superfície facial, onde fornece ramos terminais nasais internos e externos, labiais superiores e palpebrais inferiores para a pele do nariz, lábio superior e pálpebra inferior ${ }^{2,3}$.

A lesão do NIO pode ser decorrente de fraturas de órbita do tipo "blow-out", que são classificadas atualmente em dois principais tipos: puras e impuras. As fraturas puras são aquelas que ficam isoladas ao assoalho ósseo da órbita, já as fraturas impuras, são aquelas que acometem além da órbita, outros ossos faciais ${ }^{4}$. As principais causas estão associadas à atividades diárias dos indivíduos, sendo elas, coice de animais, quedas, agressões (luta, arma de fogo e outras), acidentes por automóveis, exercícios esportivos e acidentes de trabalho ${ }^{5}$. Esses traumas fazem com que fragmentos ósseos e tecidos moles invaginem-se para o seio maxilar, podendo ou não acometer o nervo infra-orbitário ${ }^{6}$.

As fraturas "blow-out" ocorrem quando há colapso do assoalho ou da parede medial da órbita, gerando perda do conteúdo ocular, conseqüente da herniação da gordura infraorbital para o seio maxilar ou para a região das células etmoidais. Esse tipo de fratura é resultante de traumas diretos sobre o globo ocular ou os rebordos orbitários, causando sua distorção e aumento da pressão intraorbitária7.

O diagnóstico dessas fraturas é realizado pelo conjunto de sinais e sintomas e acahados de imagem encontrados ${ }^{4}$. Dentre os sinais e sintomas que podem ser encontrados estão a compressão, edema, isquemia e/ou laceração do nervo por impactação dos fragmentos ósseos, além da 
enoftalmia, por abaixamento da órbita, diplopia, distúrbios sensoriais, em virtude do aumento da pressão hidráulica no interior da órbita e impacto direto ${ }^{4}$. Isto pode resultar em disestesia da pele da pálpebra inferior, da região jugal, do nariz, do lábio superior (incluindo sua mucosa), da gengiva e dos dentes do lado afetado ${ }^{4,8}$.

O tratamento das fraturas blowout é um grande desafio para o cirurgião bucomaxilofacial. Nas últimas décadas, muitos avanços vêm sendo realizados com o objetivo de restabelecer o contorno do assoalho orbitário no tratamento dessas lesões ${ }^{9}$. Diversos estudos demonstram a eficácia da reparação cirúrgica e fixação dos focos de fratura através de telas de titânio, fio de aço ou miniplacas e parafusos. A completa recuperação da sensibilidade do NIO raramente ocorre ${ }^{8,9}$.

O presente estudo objetiva medir e avaliar os diferentes comprimentos do trajeto do nervo infra-orbitário, desde o sulco infra-orbitário até a sua emergência pelo forame infraorbitário. E assim, a partir das medidas encontradas, traçar uma correlação entre elas, do ponto de vista da correção cirúrgica das fraturas de órbita do tipo "blow out".

\section{MÉTODO}

Foi realizado uma metodologia transversal, por procedimentos comparativos e estatísticos, e técnicas de medição em laboratório.

A amostra consistiu de 30 hemi-crânios secos disponíveis no Laboratório de Anatomia da Universidade 
Metropolitana de Santos (UNIMES). A análise dos complexos SIO/CIO/FIO, foi feita com auxílio de um paquímetro digital.

Para a medicão da profundidade, foi utilizada cerdas de vassoura de piaçava. Primeiramente, introduziu-se a cerda no FIO até o final da extensão do SIO, passando pelo CIO. Na mesma, foi feita uma marcação e retirada do crânio para medição com o paquímetro. Todos os dados foram anotados em uma tabela, sob a qual diversos parâmetros foram retirados e analisados nos próximos itens da pesquisa.

\section{RESULTADOS}

Dos 30 hemi-crânios analisados, diversos valores foram encontrados (Tabela 1). Sobre esses valores, foi calculado o valor mínimo, o valor máximo, a média, o desvio padrão e a variância (Tabela 2).

As medidas encontradas foram comparadas $e$ interpretadas por meio do Teste $\mathrm{t}$ de Student, que visa avaliar se há diferança significante entre os resultados de uma amostra ou entre duas amostras. Por meio desse teste foi possível obter dois gráficos da distribuição normal das medidas encontradas.

O Gráfico 1 analisa as amostras consideradas, verificando que não há diferença entre o lado direito e o lado esquerdo $(p=0,99)$. Por outro lado, o Gráfico 2 faz uma curva de acordo com todos os resultados encontrados, resultando em uma valor de $p<0,0001$, o que significa, que entre eles há diferença. 
Tabela 1. Valores do complexo FIO/CIO/SIO em milímetros (mm), comparando lado direito e esquerdo.

\begin{tabular}{|c|c|c|}
\hline Crânio & Lado Direito & Lado Esquerdo \\
\hline $\mathbf{1}$ & 17,13 & 23,14 \\
\hline $\mathbf{2}$ & 29,51 & 28,37 \\
\hline $\mathbf{3}$ & 28,44 & 36,86 \\
\hline $\mathbf{5}$ & 32,43 & 28,25 \\
\hline $\mathbf{6}$ & 26,67 & 26,97 \\
\hline $\mathbf{7}$ & 25,01 & 24,15 \\
\hline $\mathbf{8}$ & 25,02 & 37,27 \\
\hline $\mathbf{9}$ & 28,49 & 27,41 \\
\hline $\mathbf{1 0}$ & 23,06 & 21,56 \\
\hline $\mathbf{1 1}$ & 22,07 & 22,29 \\
\hline $\mathbf{1 2}$ & 26,81 & 26,39 \\
\hline $\mathbf{1 3}$ & 28,92 & 37,08 \\
\hline 14 & 26,52 & 25,33 \\
\hline 15 & 35,72 & 23,14 \\
\hline & 24,85 & 28,43 \\
\hline & & \\
\hline
\end{tabular}

Tabela 2. Valores do complexo FIO/CIO/SIO em milímetros (mm).

\begin{tabular}{|c|c|c|c|c|c|}
\hline & $\begin{array}{c}\text { Valor } \\
\text { mínimo }\end{array}$ & $\begin{array}{c}\text { Valor } \\
\text { máximo }\end{array}$ & Média & $\begin{array}{c}\text { Desvio } \\
\text { padrão }\end{array}$ & Variância \\
\hline Lado direito & 17,13 & 35,72 & 26,71 & 4,232 & 19,19 \\
\hline $\begin{array}{c}\text { Lado } \\
\text { esquerdo }\end{array}$ & 21,56 & 37,27 & 27,776 & 5,123 & 28,127 \\
\hline $\begin{array}{c}\text { Todos os } \\
\text { valores }\end{array}$ & 17,13 & 37,27 & 27,243 & 4,729 & 23,136 \\
\hline
\end{tabular}


Gráfico 1. Comparação dos valores do complexo FIO/CIO/SIO, lado direito e esquerdo.

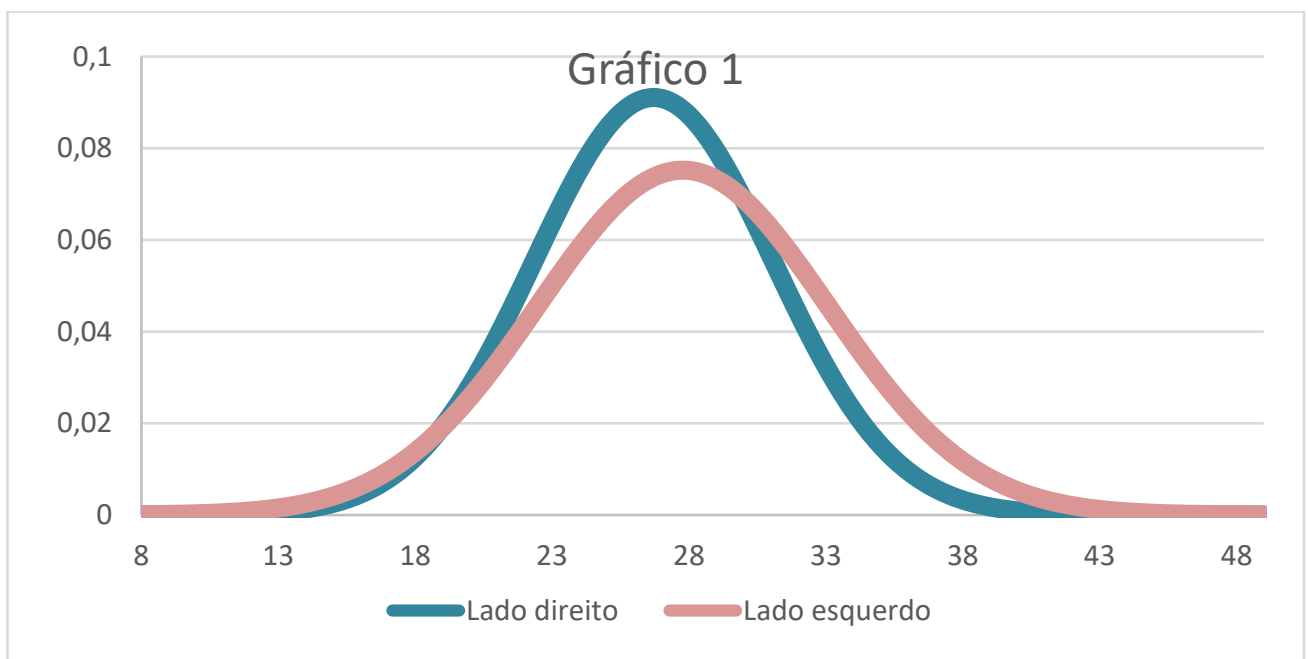

Gráfico 2. Comparação de todos os valores do complexo FIO/CIO/SIO.

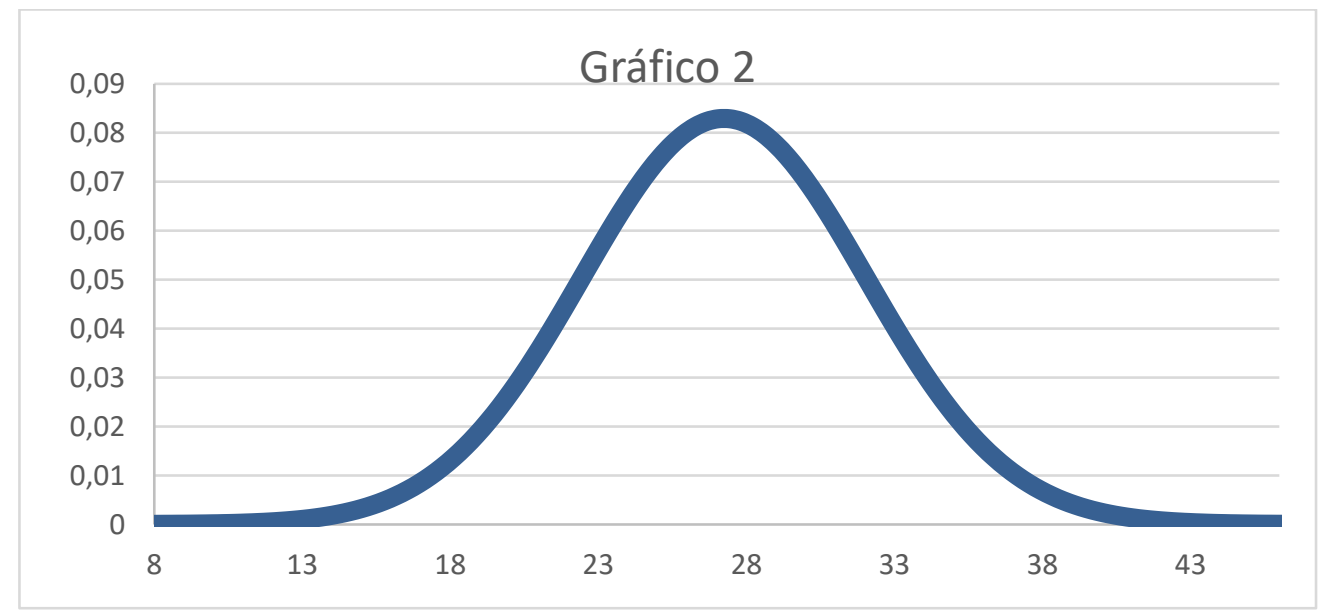

\section{DISCUSSÃO}

A análise da morfometria da região estudada, permitiu encontrar diversos resultados, que isoladamente, têm diferença significante entre eles, o que os tornam importantes no momento de uma reconstrução cirúrgica de 
uma fratura de órbita, por exemplo. Dessa forma, para um sucesso cirúrgico, torna-se importante salientar as variações encontradas nesse complexo $\mathrm{FIO} / \mathrm{CIO} / \mathrm{SIO}$, de modo que o profissional esteja sempre atento no momento de uma cirurgia. Não foram observadas diferenças entre os lados direito e esquerdo, o que evidencia que, em comparação aos lados, não há uma variação anatômica.

O nervo infra-orbital está frequentemente envolvido nas fraturas de órbita, uma vez que o complexo FIO/CIO/SIO representam pontos de fragilidade óssea, aumentando o risco da lesão neuronal. A reparação cirúrgica torna-se necessária como tratamento desses tipos de fraturas, porém no período pós-operatório, há o risco da alteração da sensibilidade do nervo, o que pode acarretar em mordedura involuntária da região mucosa ou cutânea dos lábios, perda inconsciente de líquidos pela rima bucal, ferimentos na face, ou simplesmente a sensação de adormecimento da região abaixo das pálpebras. Por esse motivo, se torna importante a avaliação da sensibilidade do NIO no pós-operatório, considerando a re-abordagem cirúrgica da reparação da órbita.

\section{CONCLUSÃO}

De acordo com os achados desse estudo anatômico, pode-se concluir que, não houve diferença significante entre os lados direito e esquerdo, e que o reconhecimento da localização o complexo $\mathrm{FIO} / \mathrm{CIO} / \mathrm{SIO}$ é de extrema importância para uma abordagem cirúrgica satisfatória e 
individualizada, a fim de tentar ao máximo preservar a função do nervo infra-orbital.

\section{REFERÊNCIAS}

1.Zide BM. Anatomia cirúrgica da região periorbital: O sistema de Zonas. Rio de Janeiro: Di Livros Editora Ltda; 2007, 144 p.

2.Shinohara AL, Coelho LAS. Estudo anatômico e tomográfico do sulco e canal infra-orbital. In: Simpósio Internacional de Iniciação Científica da Universidade de São Paulo - SIICUSP. Universidade de São Paulo; 2017.

3.Dângelo JG, Fattini CA. Anatomia humana sistêmica e segmentar. 3.ed. São Paulo: Atheneu, 2007.

4. Mororó ABG, Almeida S, Carvalho FSR, Freire Filho FW, Bezerra MF, Tavares RN. Tratamento cirúrgico de fratura orbitária blow-out pura com tela de titânio: relato de caso. Rev Odonto Bras Central 2013;22:120. https://doi.org/10.36065/robrac.v22i63.697

5. Monnazi MS, Vieira EH, Gabrielli MAC, Gabrielli MFR, Pereira Filho VA. Fraturas orbitais. Passo Fundo 2005;10:111-6.

6. Kuhnen RB, Silva FM, Scortegagna A, Cabral RJB. Fraturas de órbita: Sinais e sintomas baseados nas estruturas anatômicas envolvidas. Inter J Dentistr 2006;1:20-2.

https://periodicos.ufpe.br/revistas/dentistry/article/view/13859/1670 $\underline{6}$

7.Dantas MVM, Conte Neto N, Melo WM, Vieira EH. Fratura orbitária do tipo blow-out: relato de caso clínico. Rev Odontol 2007;36:número especial.

https://www.revodontolunesp.com.br/article/5880182d7f8c9d0a098b 4ae1/pdf/rou-36-Especial-5880182d7f8c9d0a098b4ae1.pdf

8. Fogaça WC, Alonso N, Naufal RR, Ferreira MC. Avaliação tardia da sensibilidade da face pós-fratura do complexo órbita zigomático. Rev Bras Cir Craniomaxilofac 2008;11:119-26.

http://www.abccmf.org.br/cmf/Revi/2008/Out-Dez\%202008/02\%20\%20Avalia\%C3\%A7\%C3\%A30\%20tardia\%20da\%20sensibilidade $\% 2$ 0da\%20face $\% 20$ p $\%$ C3\%B3s-

fratura $\% 20$ do $\% 20$ complexo $\% 20 \%$ C3\%B3rbito $\% 2$ zigom $\%$ C3\%A1tic o.pdf

9.Bourguignon-Filho AM, Costa AT, Ibrahim D, Blaya DS, Viegas VN, Oliveira MG. Fraturas orbitárias blow-out: tratamento com telas de titânio. Rev Cir Traumatol Buco-maxilo-fac 2005;5:35-42. http://www.revistacirurgiabmf.com/2005/v5n3/pdf\%20v5n3/v5n3.4. pdf 